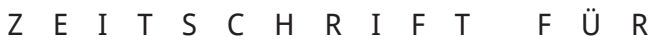

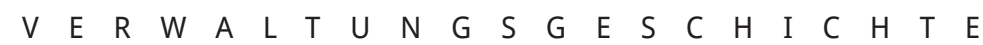

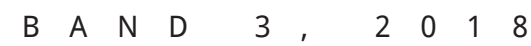

S E E I T

D O I : $10.2478 / \mathrm{ADH}$ I- $2018-0030$

\title{
Gefühlskalte Bürokratie: Emotionen im Verwaltungshandeln des frühen 19. Jahrhunderts
}

\author{
ROBERT BERNSEE
}

\section{Einleitung}

"Gefühlskalt" ist sie, wahlweise auch "menschverachtend«, "unmenschlich" oder gar ein »Monster «. ${ }^{1}$ Bürokratien derartig zu charakterisieren, ist keine Erfindung der Gegenwart, sondern hat eine lange Geschichte. Kafkas »Der Proceß«, Orwells »1984« oder Simons "The Wire" etwa eint es, die moderne Bürokratie als ein gesichtsloses oder unheimliches Etwas darzustellen - mit gefährlichen Folgen für die Menschen, die mit ihr leben müssen. ${ }^{2}$ Längst vorher kam Karl Marx zu einem ähnlichen Schluss, als er die sentfremdete Bürokratie als eine der sbürgerlichen Gesellschaft، und damit dem Kapitalismus inhärente Erscheinung deutete. ${ }^{3}$ Der Bürokratie jede Menschlichkeit abzusprechen, scheint ein beliebtes Sujet in der Moderne zu sein.

Der Topos von der 'gefühlskalten Bürokratie` lässt sich, so wird im Folgenden zu sehen sein, bereits im frühen 19. Jahrhundert beobachten. In dieser Zeit verstärkte sich die Bürokratisierung in vielen europäischen Staaten. Reformkräfte implementierten vielerorts neue Organisationsstrukturen, Rekrutierungssysteme und Formen der Vergütung, die allesamt der legal-rationalen Herrschaft zuzurechnen sind, wie sie Max Weber etwa einhundert Jahre später beschreiben sollte. ${ }^{4}$ Auf dem Gebiet des (vormaligen) Heiligen Römischen Reiches lässt sich diese Entwicklung vor allem in Preußen und einigen Rheinbundstaaten, insbesondere in Süddeutschland, beobachten. ${ }^{5}$ Die neuen Strukturelemente sollten die Verwaltungsstrukturen des Ancien Régime ersetzen, darunter den Ämterverkauf oder die Patronage. Der Reformprozess und mit ihm die Bürokratisierung waren unter anderem die Folge einer Kritik an den alten Strukturen, die in Korruptionsdebatten ab ca. 1780 formuliert wurde und sie schließlich delegitimierte. ${ }^{6}$

Mit der Bürokratisierung einher ging nicht nur ein professionalisiertes und gut besoldetes Berufsbeamtentum. Vielmehr schien es den Reformern notwendig, ein strenges Regel-, Überwachungsund Kontrollsystem zu entwickeln, um potenzielle Missbräuche seitens der Beamtenschaft zu minimieren. Diese Maßnnahmen resultierten aus der Korruptionskritik am Ancien Régime. Sie galten den Reformern nicht als problematisch, sondern zweckmäßig: Verwaltung hatte nunmehr einem abstrakten Gemeinwohl zu dienen, weshalb jedwede Verwendung öffentlicher Finanzmittel formal strikten Kontrollen unterlag. ${ }^{7}$ Staatsdiener mussten fortan offizielle Zahlungen klar belegen können; sie waren nachweispflichtig und hatten bei Unregelmäßigkeiten mit Revisionsvorgängen zu rechnen. Diese Regel- und Kontrollsysteme stellten einen Bruch mit 
der bisherigen Verwaltungskultur dar. Denn derartige Disziplinierungsmaßnahmen - zumal bei der höheren Beamtenschaft - waren bislang eher Ausnahme als Regel. ${ }^{8}$

Ziel des Aufsatzes ist es zu zeigen, dass die neuartige Bürokratie mitsamt ihren Regel- und Kontrollsystemen mit bis dahin virulenten Emotionspraktiken der höheren Beamtenschaft kollidierte. Insbesondere widersprach sie dem Konzept von Ehre, das in der alten Verwaltung als Regulator fungierte und zumal unter der adeligen Elite vorherrschte. Nach diesem Ehrkonzept kennzeichneten Kontroll- und Regulierungsmaßnahmen ein fehlendes Vertrauen seitens der Obrigkeit; sie galten den Vertretern des alten Ehrkonzepts als ein systemisch angelegtes, institutionelles، Misstrauen gegenüber deren Person. Dieser Widerspruch führte sowohl innerhalb als auch außerhalb der Verwaltung zu Konflikten und zu einer Negativdeutung des bürokratischen Systems, nämlich als ‘kaltes, seelenloses Maschinenwesen`. Als Beispiele dienen mehrere Fälle in der bayerischen Verwaltung und bürokratiekritische Autoren in der deutschsprachigen Publizistik des frühen 19. Jahrhunderts.

Um $\mathrm{zu}$ zeigen, welchen Einfluss das neuartige bürokratische System auf die Emotionspraktiken der höheren Staatsdiener nahm, sind vier Schritte notwendig: Zunächst gilt es, die hier relevanten Phänomene Emotionspraktiken, Ehre und Patronage zu erläutern. Im zweiten Teil kontextualisiere ich den Widerspruch zwischen den Ehrvorstellungen und dem bürokratischen System, indem ich auf die alte Struktur, die Kritik daran und den anschließenden Reformprozess zumal in Bayern eingehe. Im dritten Abschnitt thematisiere ich diese Konflikte im Innern der bayerischen Reformverwaltung anhand von Gabentauschpraktiken. Abschließend folgen Ausführungen zur frühen Bürokratiekritik, die zeitgenössische Reflexionen über das Verhältnis von Emotionspraktiken und dem neuen Verwaltungssystem darstellen.

\section{Emotionspraktiken, Ehre und Patronage - konzeptionelle Grundlagen}

'Gefühlskalt، impliziert einen Begriff von Emotionen, der erklärungsbedürftig ist. ${ }^{9}$ Vorliegend gelten sie als aktiver und passiver Art verbunden sind. Emotionen umfassen demnach nicht nur individuell artikulierte Zustände wie `Freude`, `Angst` oder `Wut`, sie sind darüber hinaus in bestimmte Handlungskontexte eingebunden. Damit entspricht der hier verwandte, praxeologische Zugang zu Emotionen demjenigen Monique Scheers. Sie hat beschrieben, dass Emotionen nicht von Praktiken zu trennen sind, sondern sich vielmehr darin manifestieren. ${ }^{10}$ Emotional practices definiert sie "as practices involving the self (as body and mind), language, material artifacts, the environment, and other people«. ${ }^{11}$ Monique Scheer unterscheidet vier Kategorien von Emotionspraktiken, die sich jeweils überlappen: Mobilizing practices erwecken, kanalisieren oder verstärken das Ausdrücken von Gefühlszuständen, hierzu gehört insbesondere die Nutzung von Medien. Naming practices stellen überhaupt die Gebräuche und Formen dar, Gefühle auszudrücken, während communicating practices den Austausch über bestimmte Gefühlsausdrücke meinen. Regulating practices beinhalten schließlich das normative Gefüge, beziehungsweise soziale Handlungsschemata. Letztere lassen sich als emotional styles begreifen, die das srichtige، Performieren, Interpretieren und Anwenden von Gefühlsbekundungen bestimmen. In diesem Zusammenhang weist Monique Scheer dem Bourdieu'schen Begriff `Habitus` eine zentrale Rolle zu, weil jene soziale Disposition individuelle Praktiken und deren Legitimität bestimmt. ${ }^{12}$

'Ehre`, um die es in diesem Text vor allem gehen wird, ist keine Emotionspraxis im vorgenannten Sinne. Sie fungiert nachfolgend als analytischer Begriff für einen bestimmten Typus symbolischen Kapitals nach Pierre Bourdieu. ${ }^{13}$ Ehre war zentraler Bestandteil zumal des adeligen Habitus‘ in der Frühen Neuzeit und bestimmte damit das Ensemble an Praktiken, die als legitim (oder eben nicht) für das habitusgemäße Handeln des jeweiligen Individuums galten. ${ }^{14}$ Aufgrund dessen hatte sie Konsequenzen für Emotionspraktiken. Deutlich wird dieser Umstand bei Betrachtung des Gabentauschs und damit der speziellen Praxis, die im Mittelpunkt des vorliegenden Aufsatzes steht. Er war für das symbolische Kapital von entscheidender Bedeutung. Insbesondere das Beispiel der Patronage, einer Herrschaftsform, zeitigt diesen Umstand. ${ }^{15}$ Sie basierte auf einem Gabentausch zwischen Patron und Klienten, wobei - grob gesprochen 
- Ersterer seine Mildtätigkeit oder Zuneigung durch materielle Zuwendungen kundtat, während Letzterer im Gegenzug persönliche Treue und Gefolgschaft leistete. ${ }^{16}$ Im Zusammenspiel entstand nicht nur soziale Kohärenz, etwa in Form von Vertrautheit, und eine Steigerung des persönlichen Ansehens der Beteiligten. Vielmehr lassen sich Gabentausch und damit Patronage als emotionshistorisch bedeutsame Phänomene begreifen. Dieser Befund ist nicht neu, sondern längst erkannt: Roland Mousnier war hier Vorreiter, als er die PatronKlient-Beziehungen als genuines Treueverhältnis aufgriff, mit dem eine gefühlsmäßige Verbindung zwischen den Beteiligten einherging. ${ }^{17}$ Ihm ist später vorgeworfen worden, die emotionale Verbindung zu überschätzen und dabei das eigennützige Kalkül der Akteure hintanzustellen. So etwa forderte Sharon Kettering, die gefühlsbetonte Sprache mit den sie umgebenden Praktiken der betreffenden Akteure stärker in Bezug zu setzen. ${ }^{18}$ Diese Debatte, die unter anderem auch im deutschsprachigen Raum stattfand, führte $\mathrm{zu}$ einer fruchtbaren Reflexion darüber, wie die Reichweite der Quellen einzuschätzen ist, zumal hinsichtlich der `Ernsthaftigkeit` und Beziehungstiefe der Beteiligten. ${ }^{19}$

Der Ansatz Monique Scheers ist nicht nur kompatibel mit den methodologischen Prämissen der Patronageforschung. Vielmehr liefert dieser einen Analyserahmen, mittels dessen sich Praktiken des Gabentauschs und der Patronage emotionshistorisch möglichst weitreichend erfassen lassen. Dazu ist zu verdeutlichen, welche Gefühlszustände mit diesen Praktiken verbunden sind oder, präziser formuliert, aufgrund dieser Handlungen kommunikativ verhandelt werden. >Vertrauen ‘ und ` Ehrgefühl \& fallen offensichtlich darunter. Hinzu kommen `Treue`, `Nächstenliebe` und >Pflichtgefühl beim Gebenden, aufgrund des Gebotes zur Mildtätigkeit. `Treue und sPflichtgefühl sind auch beim Gabenempfänger zu erwarten, genauso wie >Dankbarkeit،. Ein Verbot von Gabentauschpraktiken kann nun dazu führen, dass diese Gemütszustände unerreichbar werden, mit negativen Folgen für das Selbstbild der beteiligten Akteure. Und genau diese Konfliktsituation birgt Erkenntnispotenziale für die Erforschung von Emotionspraktiken. Hierzu ist hilfreich, sich zu vergegenwärtigen, dass derlei Konflikte genannten Kategorien berühren: Gabentauschpraktiken rufen Gefühlszustände hervor (mobilizing), die Geber und Empfänger offen bekunden (naming), sie erfordern reziprok eine Reaktion auf diese Gefühlsbekundungen (communicating) und unterliegen schließlich sozialen Handlungsschemata (regulating), die die Operation des Schenkens und folglich angemessene 'Gefühlsreaktionen codieren. ${ }^{20}$

Gabentauschpraktiken sind aber nicht unumstritten, sondern können als illegitim (oder illegal) bewertet werden. Dann gelten sie Zeitgenossen in vielen Fällen als korrupte Praktiken, deren Ausführung als gemeinwohlschädlich gedeutet wird. In den Aushandlungen um die Legitimität von Gabentauschpraktiken geht es damit folglich auch um Handlungsspielräume für Emotionspraktiken. Die Legitimität dieser Praktiken hing und hängt ihrerseits von den Wertmaßstäben des Referenzrahmens ab. Eine Änderung jener Werte zieht unter Umständen auch eine Delegitimierung von Gabentauschen nach sich. Und genau dies geschah in der Korruptionskritik, die eine wichtige Grundlage für die Bürokratisierung mitsamt Regel- und Kontrollinstrumenten bildete.

Im vorliegenden Fall geht es einerseits darum, wie mit dem Gabentausch verbundene Emotionspraktiken an Legitimität in Verwaltungszusammenhängen verloren gingen und wie sich der verwaltungsinterne Umgang damit ausgestaltete, zumal im Zuge der Bürokratisierung. Andererseits handelt er davon, wie betroffene Amtsträger mit dieser Entwicklung umgingen und wie sie diese bewerteten. Darauf aufbauend soll mit Blick auf die 'gefühlskalte Bürokratie` geklärt werden, welche Konsequenzen daraus für die Deutung der Bürokratisierung im Allgemeinen hervorgingen. Es handelte sich um einen Konflikt über die Legitimität von Emotionspraktiken, ja sogar emotional styles, und damit um ein historisches Ereignis, wie es Monique Scheer als besonders aufschlussreiches Anschauungsobjekt identifiziert hat. ${ }^{21}$

\section{Verwaltung vom Typ Ancien im Übergang zur Bürokratie}

Zum Kontext der alten Verwaltung und dem Niedergang derselben gehört die spezifische Zeit- 
und Raumkonstellation. Sie lässt sich für Europa um 1800 folgendermaßen beschreiben: Zunächst ist festzuhalten, dass es sich um eine politisch und gesellschaftlich dynamische Zeit handelt, weshalb sie seit Reinhart Koselleck als "Sattelzeit" bezeichnet wird. ${ }^{22}$ Kennzeichnend für Letztere sind expandierende Kommunikationsräume, zumal der Publizistik, die Folgen für gesellschaftliche Aushandlungsprozesse und Formationen sowie die politische Sprache hatten. ${ }^{23}$ Weiterhin sind die politischen Umwälzungsprozesse zu nennen, insbesondere die atlantischen Revolutionen und die napoleonische Herausforderung. Beide veränderten die politische Landkarte Europas nachhaltig. Davon war auch Bayern betroffen, das nach der Auflösung des Heiligen Römischen Reiches zum Königreich wurde und erstmals formale Souveränität erhielt, zunächst als Alliierter Frankreichs im Rheinbund. ${ }^{24}$ Noch vor der Souveränität Bayerns erfolgte eine territoriale Expansion desselben durch Mediatisierung und Säkularisation. Es waren insbesondere die Integration neuer Territorien und die Bündnisverpflichtungen gegenüber dem napoleonischen Frankreich, die in der bisherigen Forschung als Triebkräfte für die Bürokratisierung galten. ${ }^{25}$ Die alten Verwaltungsstrukturen waren nach dieser Lesart zu ineffizient, um die genannten Aufgaben zu erfüllen.

Damit wäre das bayerische Verwaltungssystem angesprochen, das sich in vielerlei Hinsicht nicht von demjenigen anderer frühneuzeitlicher Monarchien des Ancien Régime unterschied. ${ }^{26}$ Es wies jedoch einige Unterschiede $\mathrm{zu}$ modernen, bürokratischen Verwaltungen auf, die hier von Interesse sind: Zunächst lag die Verwaltung in der Vormoderne nicht in einer Hand. In den deutschen Territorien standen formal an ihrer Spitze zumeist Fürst und Landstände, die etwa in Bayern jeweils über eigene Instanzenzüge verfügten. ${ }^{27}$ Dabei verstand der Fürst seinen eigenen Verwaltungsbereich als dynastisches Eigentum, was es rechtlich auch war (Fideikommiss), und konnte sich daraus unter Beachtung gewisser Grenzen bedienen. ${ }^{28}$ Das Verhältnis zwischen Fürst und Fürstendiener war im bayerischen Ancien Régime kein sachliches, sondern ein primär persönliches: Der Fürstendiener schwor dem Fürsten lebenslange Treue und Loyalität; der Fürst sicherte ihm im Gegenzug materielle oder immaterielle des Ancien Régime einem lehnsrechtlichen oder Patron-Klient-Verhältnis. ${ }^{29}$ Dieser Umstand ist auch an der Rekrutierung erkennbar: Ämter konnten vererbt oder durch inneradministrative Patronage vergeben werden. Die Vergütung erfolgte in vielen Fällen nicht durch eine fixe Besoldung, sondern durch Naturalien oder Gebühren, sogenannte Sporteln, die die Amtsträger für Dienstleistungen von den Untertanen direkt vereinnahmten und behalten durften. ${ }^{30}$ Schließlich sind zwei Elemente der Verwaltung von Bedeutung: Einerseits gab es ein dezentrales Kassenwesen, d. h., dass die Verwaltungseinnahmen und -ausgaben vor Ort in den administrativen Einheiten getätigt wurden. Andererseits waren die Verwaltungseinheiten, zumindest auf mittlerer und höchster Ebene, kollegialisch organisiert. ${ }^{31}$ Die Entscheidungsfindung erfolgte demzufolge gemeinschaftlich durch diejenigen Fürstendiener, die in diesen Ratskollegien vertreten waren. Das Kollegialprinzip bildete inneradministrativ die soziale Hierarchie ab, da hier formell gleichrangige Adelige nicht - oder zumindest nur in abgeschwächter Form - in einem Subordinationsverhältnis zueinanderstanden. Damit wird zugleich deutlich, dass längst nicht von der Zentrale aus `durchregiert ` wurde, sondern den Praktiken vor Ort die zentrale Rolle bei der Herrschaftsausübung zukam. ${ }^{32}$

'Ehre übernahm in diesem Zusammenhang wichtige Funktionen. Sie bildete die Richtschnur für das Handeln der (zumeist adeligen) Akteure vor Ort, die danach strebten, das symbolische Kapital ihrer Personen und Familien $\mathrm{zu}$ erhöhen. ${ }^{33}$ Das Streben nach Ehre prägte den Habitus der Amtsträger und wirkte innerhalb als auch außerhalb der Verwaltung. Nach innen reichte es deshalb, weil der Fürstendiener seinem persönlichen Treueversprechen zum Fürsten entsprechend handeln musste, um seine persönliche Ehre oder diejenige seiner Familie nicht zu gefährden. Gleiches galt für den Fürsten, dem - selbst Mitglied eines Adelshauses - ebenfalls Pflichten daraus erwuchsen. Nach außen wirkte das Streben nach Ehre insofern, als die bayerischen Amtsträger selbst in Patron-KlientBeziehungen $\mathrm{zu}$ lokalen Personen oder Familien eingebunden waren. Die Rechte und Pflichten, die aus diesen Beziehungen resultierten, bestimmten das ehrgemäße Handeln des Amtsträgers und seiner Familie. Es hatte also Konsequenzen für Emotionspraktiken. 
Die Gabentauschpraktiken zwischen Amtsträger und Lokalen aktivierten Gefühlsbekundungen für das Gegenüber und vice versa. Dieses Streben nach Ehre, freilich auf einer höheren Ebene, lag auch beim Fürsten vor und provozierte auch hier beidseitig Bekundungen der Treue oder Dankbarkeit. Es genoss also höchste Priorität. Fürstendiener handelten dann 'korrupt`, wenn sie die persönliche Treue zum Fürsten oder sein Vertrauen zu persönlichen Zwecken missbrauchten. Ein Abweichen von dem sozialen Handlungsschema, insbesondere von treuebekundenden Aktionen, galt als missbräuchlich. Mit anderen Worten waren emotional styles zutiefst mit governance styles verschränkt.

Das vorgenannte Verwaltungssystem geriet ab den 1770er-Jahren in eine nachhaltige Legitimitätskrise. Es hatte schon zuvor je nach Interessenlage der Herrschaftsträger entweder Kritik an Ämterkauf, Ämtervererbung und Patronage gegeben. ${ }^{34}$ Doch nun erfuhren die Verwaltungsstrukturen des Ancien Régime eine grundsätzliche und übergreifende Kritik, zumal in der Publizistik. Sie wurden als endemisch korrupt wahrgenommen. 'Korrupt` meint hier konkret, dass die Vergütung durch Sporteln und der Amtserwerb über Kauf, Erbe oder Patronage nun als Missbrauch und Bereicherung der beteiligten Personen - gedeutet wurden. $^{35}$ >Missbräuchlich ‘ wurden diese Praktiken deshalb, weil dem neuen Korruptionsverständnis eine andere Gemeinwohlvorstellung zugrunde lag als zuvor. Zentral war nun die Vorstellung des Wohls eines abstrakten Gemeinwesens, welches aus formal gleichberechtigten (männlichen) Bürgern bestand. In Bayern ging die Korruptionskritik von einer jüngeren, akademisch gebildeten Fürstendienerelite aus, die sich unter anderem $\mathrm{zu}$ diesem Zweck in Geheimbünden organisiert hatte. Nichtsdestotrotz überlebten die alten Strukturen bis zur Jahrhundertwende. Erst nach dem Regentenwechsel 1799 kam die jüngere Beamtengeneration in entscheidende Verwaltungspositionen, mit ihr vor allem Maximilian Montgelas, engster Vertrauter des Kurfürsten und späterer Minister, der sich zuvor über mehrere Denkschriften an der Korruptionskritik wider das Ancien Régime beteiligt hatte.

Mit dem Regierungswechsel verschärfte sich der Ton gegen die alten Strukturen weiter. Es kam zudem sehr rasch zu einer Umgestaltung des Verwaltungssystems.
Nach und nach erfolgte die formale Implementierung von einzelnen Elementen einer bürokratischen Struktur: ${ }^{36}$ Sporteln wurden durch fixe Besoldungen ersetzt, die Verwaltungsorganisationen neu und funktional gestaltet, die Diensteintrittskriterien bei höheren Posten auf akademische Abschlüsse beschränkt. In Form der Dienstpragmatik erfolgte 1805 das erste, moderne Beamtengesetz im deutschsprachigen Raum, in dessen Folge sich die persönliche Dienertreue des Fürstendieners zur sachlichen Diensttreue des Staatsdieners umwandelte. Weiterhin wichtig waren die Abschaffung des Kollegialprinzips und die Durchsetzung einer monokratischen Entscheidungsstruktur in sämtlichen Teilen der Verwaltung, womit klare Hierarchien innerhalb der Verwaltung entstanden, die zudem von den ständischen abweichen konnten. Und schließlich galt für Staatsdiener ein umfassendes Korruptionsverbot modernen Zuschnitts. Ihnen war es untersagt, Gaben von außerhalb der Verwaltung anzunehmen oder inneradministrativ Patronage $\mathrm{zu}$ betreiben. Derlei Übertritte wurden strafrechtlich normiert und sanktioniert, wie es vorher nur ansatzweise der Fall gewesen war. ${ }^{37}$

Hinzu kamen umfassende Kontrollmechanismen, die die Regulationsfunktion von Ehre weitgehend ersetzten. Sie waren eine Folge des zentral organisierten Kassenwesens, das das bisherige ersetzte, und damit Ausdruck einer verstärkten Arbeitsteilung innerhalb der Verwaltung. ${ }^{38}$ Es entstand ein Finanzministerium, das die meisten Ein- und Ausgaben kontrollierte. Dieses Ministerium stand in einer Budgetbeziehung zu den übrigen, die ihrerseits derlei Beziehungen mit untergeordneten Behörden aufrechterhielten, und wies Finanzmittel an diese zu. Damit waren andere Kontrollmechanismen notwendig geworden, da die einzelnen Verwaltungseinheiten finanziell viel stärker miteinander verflochten waren und diese Finanzströme dokumentiert werden mussten. Mit anderen Worten hatte sich in der Verwaltung eine Gabenkultur und -struktur eingeschrieben, die sich von der vorherigen unterschied: Amtsträger erhielten ein festes Gehalt und eine Altersversorgung, waren aber nun verpflichtet, budgetwirksame Aktivitäten anzuzeigen und zu belegen; im Falle von Unregelmäßigkeiten setzte die Zentrale routinemäßig eingerichtete Untersuchungskommissionen ein. 
Beamte galten als rationale Nutzenmaximierer, ihr egoistischer Drang nach Materiellem bedurfte einer Disziplinierung. Es entstand also etwas, das sich als ein organisationsinternes, institutionalisiertes oder, kurz, institutionelles Misstrauen deuten ließs.

\section{Konflikte um Gabentauschprak- tiken im Innern der Verwaltung}

Der Reformregierung in Bayern war es rasch gelungen, das bürokratische Organisationsgefüge zu entwerfen und in rechtliche Form zu gießen. Dieses Gefüge in die Praxis umzusetzen, bildete eine Herausforderung ganz eigener Art. Wie würden die Beamten und Verwalteten reagieren? In diesem Abschnitt wird zu sehen sein, dass beide das neue Verwaltungssystem nicht unbedingt als 'Segen ‘ für sich empfanden. Im Gegenteil, es stand den bis dahin üblichen Emotionspraktiken, wie sie bisher den administrativen Alltag bestimmten, entgegen. Die Ausführungen erfolgen anhand mehrerer Fallbeispiele: Zentral ist der Fall um den Grafen Reisach, die kleineren Fälle zu dessen Bruder, zu Mertz und Gropper sollen die daraus gewonnenen Erkenntnisse weiter vertiefen.

Graf Karl August von Reisach-Steinberg (1774-1846) ist par excellence ein Repräsentant der salten Elite und damit auch der alten Praktiken. Er entstammte einer aufstrebenden Familie, die erst 1790 in den Reichsgrafenstand aufgestiegen war. Ausgestattet mit derlei symbolischem Kapital, sein Pate war zudem der Herzog von Pfalz-Zweibrücken, startete Reisach eine vielversprechende Karriere als Fürstendiener: Sein Vater, selbst Oberjagdamtkommissär in Pfalz-Neuburg, übertrug ihm per Ämtervererbung die eigene Stelle. Reisach, gerade 20-jährig, gelang es rasch, durch Kauf und Heirat innerhalb der Verwaltung aufzusteigen. ${ }^{39}$ So etwa tauschte er das ehemalige Amt seines Vaters gegen einen lukrativeren Posten ein und zahlte dem ehemaligen Amtsinhaber eine entsprechende Ablösesumme. Seine Heirat mit einer Tochter eines örtlichen Beamten (`Pfleger`) versprach die Aussicht, nach dem Ableben desselben weiter aufzurücken. ${ }^{40}$

Diese Praktiken scheinen Reisach als karriere- oder prunksüchtigen Mann zu enttarnen. ${ }^{41}$ Doch eine solche musste er derlei Praktiken vollziehen, wenn er denn sein symbolisches Kapital steigern - oder auch nur erhalten - wollte. ${ }^{42}$ Höhere Ämter bedeuteten größeres Prestige und erlaubten einen größeren Zugriff auf materielle Ressourcen. Letzteres war insofern notwendig, als Reisach über eigene Klienten verfügte. Mit denen stand er in Gabentauschbeziehungen und musste für deren materielles Auskommen - und dasjenige ihrer Familien - sorgen. Als Patron war er verpflichtet, seine Klienten ökonomisch zu unterstützen, um im Gegenzug deren Treue zu erhalten. Gabentauschpraktiken verknüpften Reisach und seine Klienten als Personen miteinander, sei es über Körper, Artefakte oder Sprache; sie begründeten folglich eine beiderseitig wirksame Nahbeziehung. Gefühlsbekundungen waren elementarer Teil dieser Interaktion.

Die Ankunft der Reformregierung tat seiner Karriere keinen Abbruch, sondern das Gegenteil war der Fall: Reisach war Mitglied der Landschaft und deshalb für die neue Regierung höchst interessant. Die Landschaft vertrat die Landstände und war damit (noch) an der Herrschaftsausübung Altbayerns beteiligt. ${ }^{43}$ Das neue Regime bedurfte ihrer Unterstützung, um die territoriale Herrschaft über Bayern zu sichern. Keinen Abbruch daran tat die Korruptionskritik, die sich gegen die Verwaltungsstrukturen des Ancien Régime richtete und sie maßgeblich delegitimierte. ${ }^{44}$ Hinzu kam, dass sich Bayern insbesondere zwischen 1803 und 1806 territorial erheblich ausdehnte und deshalb auf erfahrene Personen angewiesen war. Reisach stieg nach dem Regierungswechsel auf, erreichte die Position des Generalkommissärs und damit die höchste auf mittlerer Verwaltungsebene seit den Reformen 1808. Tätig war er zunächst im Lechkreis, danach im Illerkreis, die beide zwei Jahre zuvor als administrative Einheiten entstanden waren. Letzterer umschloss Vorarlberg und das westliche Tirol, also Territorialgewinne Bayerns aus dem Frieden von Pressburg. Der Illerkreis darf als eine der unruhigen Gegenden gelten, da er besonders von den Aufständen der Vorarlberger und Tiroler gegen die bayerische Herrschaft betroffen war. ${ }^{45}$

Schon vor seiner Beförderung zum Generalkommissär, nämlich 1805, kam es zu Unregelmäßigkeiten in der Amtsführung. Reisach hatte offenbar aus Kassenbeständen unzulässig Beträge entnommen, um damit seinen Untergebenen höhere 
Diäten und Gratifikationen zu bezahlen. Er leistete damit seiner Pflicht als Patron Folge. In der Zentrale hatte man diese Unregelmäßigkeiten registriert, zumal in der Belegführung. Eine Untersuchungskommission wurde eingesetzt; doch Reisach widersetzte sich der Überprüfung. Er wertete dieses Vorgehen als Angriff auf seine Ehre und beschwerte sich darüber direkt beim König, der daraufhin einlenkte und die Gratifikationen genehmigte. ${ }^{46}$ Nur wenig später folgte eine weitere Untersuchung: Reisach hatte im Verlaufe seiner Amtstätigkeit Douceurs - also Geschenke an verbündete französische Soldaten geleistet und damit eigenmächtig über Finanzmittel verfügt. Als Repräsentant der Regierung praktizierte er demzufolge Gabentauschpraktiken mit Verwaltungsexternen, um mit diesen persönliche Nahbeziehungen aufzubauen. Die Zentrale übte wiederholt Kritik daran, die Reisach als einen Angriff auf seine Ehre beklagte. In einem persönlichen Brief von 1808 an den zweiten Mann im bayerischen Staat, Minister Montgelas, heißt es, dass er

alles geleistet habe, was man von einem treuen Staatsdiener, und von dem Mann von Ehre fodern

kann, und daß es traurig ist, wenn man für [...] Arbeit und Mühen noch seinen guten Namen aufs Spiel gesetzt [bekommt]. ${ }^{47}$

Dem König empfahl er gar die Entlassung seiner Person, sofern kein Vertrauen mehr bestünde. An diesem Zitat wird deutlich, wie sehr Reisach die Untersuchungskommission eo ipso als persönliche Kränkung empfand oder, präziser, eine solche offen bekundete. Er kommunizierte eine Verletzung seines Ehrgefühls und die Empfindung, dass ihm Misstrauen entgegengebracht wurde, sowohl seitens des ersten Ministers als auch des Königs. Zugleich bekundete er seine Treue und seine Traurigkeit über den Umgang mit seiner Person. Im Umkehrschluss heißt dieser Umstand, zumal er die eigentlichen Taten gar nicht bestritt, dass er die Qualität seiner Praktiken gänzlich anders bewertete als die Münchner Zentrale. Nach seiner Argumentation waren die Gabentauschakte mit Verwaltungsexternen genauso legitim, wie die Patronagebeziehungen zu seinen Untergebenen - und dem König. Persönliche nicht nur Bestandteil von Verwaltungspraktiken sein, sondern waren sogar ein wichtiges Ziel derselben. Der König entschied sich interessanterweise nicht nur gegen eine Entlassung Reisachs, sondern beförderte ihn sogar zum Generalkommissär. Damit reagierte er auf Reisachs offene Gefühlsbekundungen, wie man es von einem Patron erwarten durfte - mit Handlungen, die Respekt und Vertrauen implizierten. Der König folgte damit dem üblichen emotional style und bestätigte mit seiner Handlung dessen Legitimität in Verwaltungszusammenhängen.

Angesichts dieser Entwicklung ist kaum verwunderlich, dass Reisach sein Vorgehen nicht veränderte. Insbesondere behielt er sein freigiebiges Gebaren gegenüber Untergebenen bei, ohne es sich von der Zentrale genehmigen $\mathrm{zu}$ lassen. Auch im Zusammenhang mit Verwaltungsexternen agierte er nicht anders: Nach eigenem Ermessen interagierte er mit einheimischen Eliten, d. h., er unterhielt Gabentauschpraktiken mit ihnen im Namen der Krone. Dabei handelte es sich durchaus um einen traditionellen Umgang mit neu erworbenen Territorien. Als ranghöchster Staatsdiener - quasi Gouverneur - und zudem hochadelig war ein solches Agieren für Reisach habitusgemäß und damit legitim. Für die bürokratische Zentrale galt dieser Umstand längst nicht mehr, sie monierte 1810 erneut seine Handlungen. Reisachs Ehrverweise nützten ihm diesmal nichts; er hatte einen Teil der entstandenen Kosten zu erstatten. Und es kam noch schlimmer für ihn: Die nun eingesetzte Untersuchungskommission stellte fest, dass er Unterschlagungen in enormer Höhe während seiner Tätigkeit als Generalkommissär vorgenommen hatte. Offenbar hatte er Schuldverschreibungen an gutgläubige Bürger ausgegeben, ohne die vereinnahmten Gelder weiterzureichen. Der Rechenschaft entzog sich Reisach diesmal, indem er Bayern verließ und über Sachsen nach Preußen flüchtete. ${ }^{48}$

Reisachs Praktiken waren, abgesehen von dieser exorbitanten Unterschlagung, kein Einzelfall, sondern kennzeichnend für eine Verwaltungskultur, in der administrative Praktiken, zumal der Gabentausch, zugleich einen bestimmten emotional style codierten. Diesen Befund legen auch andere Fälle nahe, etwa derjenige Franz von Groppers. Jener war Kanzleidirektor des Illerkreises und bekleidete damit 
ebenfalls eine ranghohe Position. Gropper, so lautete 1815 das Ergebnis einer Routineüberprüfung, war mit Diäten und Reisegeldern offenbar sgroßzügig، umgegangen. ${ }^{49}$ Bemerkenswerter ist aber etwas anderes: Im gleichen Jahr fragte er seinen Vorgesetzten, ebenerwähnten Minister Montgelas, ob er eine "goldene einfache Tabattiere« behalten dürfe. Dieses Geschenk hatte er von der lokal ansässigen Gräfin Waldbott-Bassenheim und deren Mann, »als Andenken ihrer Freundschaft « erhalten, für eine Gefälligkeit, die außerhalb seiner Amtstätigkeit gelegen hätte. ${ }^{50}$ Montgelas verwehrte ihm die Annahme und verwies darauf, dass die vermeintliche `Gefälligkeit` eine reguläre Amtshandlung gewesen sei. ${ }^{51}$ Anders gesagt, sein Gehalt war die einzige Belohnung, die Gropper dafür erwarten konnte, persönliche Nahbeziehungen zu Verwalteten - etwa in Form von Freundschaften - durften auf diese Weise nicht entstehen. Der Fall zeigt eindrücklich, wie unsicher sich Beamte ob der Zulässigkeit bestimmter Praktiken waren. Zudem gibt er darüber Auskunft, dass auch Außenstehende Gabentauschpraktiken initiieren konnten. Sie waren in diesem Fall ein Ausdruck der Dankbarkeit und eine Ehrerbietung, die damit zu Bekundungen von Gemütszuständen bei Gebenden und Empfängern führen konnte und einem sozialen Handlungsschema folgte. Tatsächlich waren sgoldene Tabakdosen gängiges Geschenk, zumal im diplomatischen Verkehr des 18. Jahrhunderts, um Zuneigung auszudrücken. ${ }^{52}$ Gropper befand sich in der schwierigen Lage, dass das damit verbundene soziale Handlungsschema für Emotionen demjenigen der bürokratischen Verwaltung, hier repräsentiert von Montgelas, widersprach. Letztere schrieb Gropper vor, wie die Antwort auf derlei Gaben auszusehen hatte (sofortige Rückgabe). Amtsträger hatten sich ganz im Sinne ihres Dienstes am abstrakten Gemeinwohl zu verhalten; persönliche Nahbeziehungen waren davon auszuklammern, weil sie eine praktische Umsetzung dieses Dienstes potenziell gefährdeten.

Ungeachtet dieses Falls lohnt es sich, auf die vermeintlichen sEhrverletzungen` Reisachs zurückzukommen. Gegen die hier verfolgte Argumentation ließse sich einwenden, die Betroffenen würden Ehrverletzungen nur taktisch einsetzen, um von der eigentlichen Tat abzulenken. Demnach handele es sich dabei lediglich um einen Wechsel des Kommunikationsmodus und nicht um die sechter Reflexion von Gemütszuständen. Dieser Einwand lässt sich theoretisch nicht zurückweisen: Der Verweis auf eine Ehrverletzung war schwerwiegend. Er konnte seitens der Akteure taktisch eingesetzt werden, um persönliche Angriffe abzuwehren. Allerdings ginge eine solche Interpretation letztlich von einer Binarität von emotional/rational aus, da sie eine srationales Instrumentalisierung vermeintlicher Gefühlszustände seitens der beteiligten Akteure annimmt. Abgesehen von der methodologischen Problematik, diese Binarität ex ante anzunehmen oder überhaupt eine sechte، Reflexion eines Gefühlszustandes historiografisch $\mathrm{zu}$ ermitteln, sei an das hier verfolgte Erkenntnisziel erinnert: Es geht darum, die Konflikte um den Gabentausch - und damit Patronage - als Aushandlung über die Zulässigkeit von Emotionspraktiken im Sinne Monique Scheers zu begreifen und die Folgen dieser Aushandlung für die beteiligten Akteure einzubeziehen. Wie der kommunizierten Verletzung des Ehrgefühls und der damit verbundenen Demütigung seitens beteiligter Akteure begegnet werden konnte, vermitteln die nachfolgenden Fälle. Nachdem ein 'Ehrverlust، aufgrund eingeleiteter Untersuchungsvorgänge eingetreten war oder wenigstens einzutreten drohte, töteten sich die Protagonisten in beiden Fällen selbst. ${ }^{53}$ Der erste betrifft Reisachs Vorgänger im Illerkreis, Ludwig Balthasar von Mertz. Er geriet 1809 in das Fadenkreuz der Ermittlungen, weil er offenbar eigene Klienten in Position gebracht und Geschenke von den Landständen angenommen hatte. Mertz bestritt die Gabentauschakte nicht und versicherte, dabei nur die Interessen des Fürsten und Landes verfolgt zu haben. Wie in den anderen Fällen deutete er seine Handlungen als Ausdruck seiner Treue gegenüber den Fürsten und als Ehrerbietung gegenüber den Landständen. Als sich die Ermittlungen hinzogen und sich ein wohl für ihn ungünstiger Ausgang abzeichnete, ertränkte er sich im Lech - wohl, um dem drohenden Ehrverlust $\mathrm{zu}$ entgehen. ${ }^{54}$ Der zweite Fall dreht sich bemerkenswerterweise um Reisachs Bruder, Johann Adam von Reisach-Steinberg (1765-1820), der ein hoher Lokalbeamter im schwäbischen Monheim war. Er beschwerte sich vehement bei der zentralen Bürokratie, nachdem diese die bisher üblichen Sporteln einkassiert 
und durch fixe Besoldungen ersetzt hatte. ${ }^{55}$ Reisach verwies darauf, dass er aufgrund des verringerten Einkommens nicht mehr den Versorgungspflichten gegenüber seiner Familie und seinen Unterbediensteten nachkommen konnte. Er machte also deutlich, dass er sein Pflichtgefühl gegenüber verbundenen Personen verletzt sah und ihnen seine Dankbarkeit nicht ausdrücken konnte. Die bürokratischen Reformen schränkten folglich seinen Spielraum für Gabentauschpraktiken ein und damit seine Möglichkeiten, ein ehrbares Verhalten an den Tag zu legen. Seine Beschwerde verhallte folgenlos. Wenig später kam es zu Kassenunregelmäßigkeiten, scheinbar hatte er seine Praktiken stillschweigend beibehalten, womöglich seinerseits als Antwort auf die Treuebekundungen seiner Klienten. Den eingeleiteten Untersuchungen und dem damit verbundenen Ehrverlust entzog sich Reisach durch Selbsttötung. Beide Beispiele zeigen, wie sich die Handlungen ausgestalten konnten, nachdem die Protagonisten auf ein verletztes Ehrgefühl und entgegengebrachtes Misstrauen hingewiesen hatten. Die Protagonisten werteten die Maßnahmen der Bürokratie als schwere Demütigungen, denen sie den eigenen Tod offenbar vorzogen.

Die hier vorgestellten Fälle bayerischer Spitzenbeamter belegen, so lässt sich festhalten, dass der bis dahin übliche emotional style in Konflikt mit den bürokratischen Reformen geriet. Im Zentrum standen dabei Gabentauschpraktiken, die als Medium für Bekundungen der Dankbarkeit, des Pflichtoder Treuegefühls fungierten und einem sozialen Handlungsschema folgten. Der Gabentausch diente letztlich dem Aufbau und Erhalten von Ehre. Dieser Umstand galt nicht nur für die Amtsträger, sondern auch für deren Klienten und Verwaltungsexterne. Die bürokratischen Maßnahmen sollten bestimmte, nun 'korrupter Gabentauschpraktiken unterbinden helfen und eine ordnungsgemäße Durchführung administrativer Praktiken gewährleisten. Sie zielten keineswegs darauf ab, Verwaltungsmitglieder $\mathrm{zu}$ demütigen. Dennoch trat dieser Umstand ein: Die Betroffenen deuteten die Kontrollmechanismen als institutionelles Misstrauen und damit als ehrenrühriges Instrument, weil es ihrem Handeln zuwider stand, das dem dominierenden emotional style

\section{Zur Aushandlung von emotional styles in der frühen Bürokratiekritik oder: die Konstruktion der sgefühlskalten Bürokratie`}

Diese Konflikte um emotional styles, wie sie sich im Innern der Verwaltung ereigneten, hinterließen eine Spur in der publizistischen Debatte. Sie führten, wie im Folgenden zu zeigen sein wird, zu einer Deutung der Bürokratie als einem Ort, der aufgrund seiner Negation von bisher etablierten Emotionspraktiken unmenschlich geworden sei. Zeitgleich mit der Bürokratisierung entstand in der Publizistik eine Kritik daran sowie dem Verwaltungssystem als solchem. ${ }^{56}$ Diese Bürokratiekritik hatte ihre Vorläuferinnen bereits im 18. Jahrhundert, als die fürstliche Verwaltung zumal mit dem Aufkommen der Kameralistik an Dynamik und Schriftlichkeit gewann. Die Rede war vom 'Staat als Maschine.$^{57}$ Mit den Reformprozessen um 1800 erreichte diese Kritik eine neue Qualität. Eine ganze Reihe deutschsprachiger Autoren kritisierte Bürokratie und Bürokratisierung. Nachfolgend geht es darum zu zeigen, dass der Topos von der sgefühlskalten Bürokratie، zentral für diese Kritik war, um die neue Verwaltung als solche zu diskreditieren. Berücksichtigung finden die Schriften von August Rehberg, Adam Müller, Ernst von BülowCummerow und einem alten Bekannten, nämlich ebenerwähntem Karl August von Reisach.

Den Schriften war der Vorwurf gemeinsam, dass die Beamtenschaft durch die Bürokratisierung systematisch >verdorben ‘ wurde. Laut August Rehberg (1757-1836) erziehe das neue System jeden Beamten gewissermaßen zu einem »Werkzeug « innerhalb einer wohlgeordneten "Maschine ${ }^{58}$ Sie sehen "in ihrem Berufe nur ein Gewerbe« - ein Umstand, der letztlich $\mathrm{zu}$ rein eigennutzorientierten und ausschließlich pflichtmäßigen Handlungen des Beamten führe.59 Der preußische Publizist Adam Müller (1779-1829) charakterisierte die neue Verwaltungsstruktur als ein »todtes Räderwerk «, deren Mitglieder sich nur im KleinKlein verlören und sich zudem nur um die Erhöhung der eigenen Besoldung scheren würden. ${ }^{60}$ Die Autoren kritisierten, dass die Staatsdiener nun zwar akademisch gebildet seien, gewissermaßen "Theoristen ${ }^{61}$ Indes 
fehle es ihnen an Einfühlungsvermögen sowie an Kenntnissen über lokale Gegebenheiten - und damit der Fertigkeit, vor Ort einen echten Dienst am Gemeinwesen leisten zu können. ${ }^{62}$ Die bürokratischen Rekrutierungsund Vergütungsstrukturen, so die Autoren einhellig, hätten die Beamtenschaft also zum Schlechteren verändert. Sie bestünde aus karriereorientierten Berufsbeamten, die kein Ehr-, Treue- oder Pflichtgefühl mehr kannten. Das System als Ganzes sei folglich dem moralischen Niedergang ausgesetzt.

An diesem Umstand änderten nach Auffassung der Autoren auch die bürokratischen Schutzmechanismen nichts, die hier bislang analytisch unter das sinstitutionelle Misstrauen ' gefasst wurden. Im Gegenteil, sie seien nur wenig schlagkräftig: „Vergebens ordnet man Revisionen und SuperRevisionen an, und häuft Controlen auf Controlen«, heißt es bei Rehberg, doch Missbräuche der Beamten seien damit keineswegs zu verhindern. ${ }^{63}$ Noch besser auf den Punkt bringt es ein Publizist aus Preußen, Ernst von Bülow-Cummerow (1775-1851). Er kritisiert, wie auch die anderen Autoren, die als massiv wahrgenommene Expansion der fürstlichen Verwaltung, zumal der sinnere Geist، dieser Verwaltung nur Nachteile nach sich ziehe:

\section{Je mehr regiert wird, je mehr Beamten sind nöthig; damit diese jedoch keinen Mijbrauch ihrer Gewalt machen, setzt man andere an, die diese controlliren, dadurch verschlimmert sich die Sache, denn nun sind schon zwei Behörden zu controlliren, dies fordert die dritte, so geht's immer fort [...]. ${ }^{64}$}

Bülow zufolge ziehe das sinstitutionelle Misstrauen eine Reihe von Kontrollen nach sich; es unterliege einer Art Rekursion, dessen Auflösung mit den Mitteln der bürokratischen Verwaltungsstruktur logisch nicht möglich war. Damit widerspricht Bülow im Übrigen den Kritikern der alten Verwaltungspraktiken, die die Bürokratie als Lösung für die endemische Korruption sahen. Denn bürokratische Kontrollmechanismen könnten Missbräuche niemals vollständig eliminieren, würden jedoch zwangsläufig zu einer permanenten Ausdehnung der Verwaltung führen. Bülow vertrat damit eine allzu vertraute Argumentation, nach der Bürokratien einer ständigen Expansion ausgesetzt sind - obwohl die Verwaltungen der Reformzeit, etwa in Preußen, im frühen 19. Jahrhundert keineswegs außergewöhnlich stark wuchsen - im Gegenteil. ${ }^{65}$

Die Autoren kritisierten nicht nur die Verwaltungsstrukturen mit ihren impliziten Annahmen und kontrollierenden Mechanismen. Ein wichtiges Argument war die `kalte Rationalitätı des neuen Verwaltungssystems, die bereits bei Rehberg und Müller angeklungen war. Die Schriften von keinem Geringeren als Karl August Reisach illustrieren diesen Sachverhalt in hohem Maße: Reisach war nach seiner Flucht aus Bayern zunächst in Sachsen und dann vor allem in Preußen aktiv. Er gelangte in den engeren Kreis um den preußischen Reformer Karl vom und zum Stein (1757-1831). Jener hatte ein publizistisches Netzwerk aufgebaut, dessen Mitglieder gegen Napoleon und seine Verbündeten skandierten. ${ }^{66}$ Unter Steins Protektion verfasste Reisach einige Pamphlete, in denen er das bayerische Reformregime als moralisch verdorben charakterisierte. Es entwickelte sich ein regelrechter Flugschriftenkampf, denn bayerische Autoren veröffentlichten einige offiziöse Schriften, in denen sie die Verfehlungen Reisachs als Staatsdiener den Vorwürfen entgegenhielten. ${ }^{67}$ Im Detail ist diese Auseinandersetzung hier von untergeordnetem Interesse; bemerkenswert sind indes die Beschreibungen Reisachs, wie sich die Kultur der nunmehr bürokratischen Verwaltung ausgestalten würde. Beispielhaft dafür ist folgendes Zitat:

\section{[L]osreißen sollte sich der Staatsdiener von den Verhältnissen, die ihn bis dahin so wohlthätig an seine Mitbürger geknüpft hatten. Zur Maschine geworden, die säet, schneidet, und bis auf das letzte Körnchen rein ausdrischt, sollte er kein Herz, kein Gefühl, keinen Freund, keinen Acker mehr haben, und sich von einem Ort zum anderen transportiren lassen, um überall neuerdings als Wahrzeichen aufgestellt zu werden, vor dem alles herzliche Gefühl, alles Vertrauen erkalten mußte. ${ }^{68}$}

Reisach beschreibt die neue Verwaltung als einen Ort, an dem persönliche Beziehungen oder emotionale Bekundungen unerwünscht - oder gar unmöglich sind. Die Administration ist nach seiner Auffassung ein Ort geworden, der den Menschen feindlich ist, obwohl er ihnen dienen soll. Nimmt man diese Aussagen 
ernst und verknüpft sie mit den Befunden zu seinen Praktiken, dann wird klar: Reisach versucht hier, seine Gabentauschakte zu Untergebenen und lokalen Eliten nicht nur zu legitimieren. Er zielt vielmehr darauf, die Praxis als zentrale Kategorie administrativen Handelns $\mathrm{zu}$ etablieren. Nach seiner Auffassung waren diese Emotionspraktiken notwendig, um das Vertrauen der Untertanen $\mathrm{zu}$ gewinnen und persönliche Bande $\mathrm{zu}$ ihnen zu knüpfen. Gabentauschakte waren demnach Ausdruck eines emotional style, den Reisach zugleich als unabdingbar für eine sgute Regierungsführung، verstanden wissen will. Anders gesagt, es sei für die Erhöhung des sallgemeinen Besten ‘ - mithin des obersten Verwaltungszwecks - zwingend notwendig, soziale Handlungsschemata zuzulassen, die persönliche Bindungen zwischen Amtsträgern und Untergebenen erlaubten und förderten. Ähnliche Auffassungen lassen sich bei Müller und Rehberg finden: Ersterer sah in der ,Vergötterung des Sächlichen statt Persönlichen eine zentrale Ursache dafür, dass sich die zeitgenössische Verwaltung im Niedergang befinde. ${ }^{69}$ Letzterer zweifelte die Aussagekraft statistischer Erhebungen an, die für Entscheidungen innerhalb von Bürokratien zentral werden sollten. Tatsächlich hatten statistische Erhebungen durch die Gründung entsprechender Organisationen um 1800 an Auftrieb gewonnen. ${ }^{70}$ Rehberg bestritt vehement, dass die jüngst etablierte Erhebungs- und Datensammlungspraxis, nämlich die "Statistik", sich überhaupt am "gemeinen Wesen" orientieren würde. ${ }^{71}$

Die bisherigen Ausführungen deuten an, was diese Autoren der von ihnen geschmähten Verwaltung positiv entgegensetzten: Die Antwort lautet schlicht - Ehre und Tugend. Sie forderten ziemlich einhellig, dass jede Verwaltung tugendhafter Personen bedarf, um wirkungsvoll dem Gemeinwohl dienen zu können. Gemeint waren tugendhafte Adelige: Reisach hob hervor, dass insbesondere adelige Beamte mit lokalen Kenntnissen notwendig waren und kein akademisch geschultes Personal. ${ }^{72}$ Nach Bülow hätte »der Geist der alten Institutionen bewahrt werden « müssen und nicht, wie geschehen, während des Reformprozesses verschwinden dürfen. ${ }^{73}$ Die Autoren sprachen sich dabei für eine Erneuerung des Adels aus, der zu seinen ursprünglichen Eigenschaften zurückkehren sollte, vor für Rehberg, der sich für die Konstituierung einer "DienstAristokratie« ausspricht. ${ }^{74}$ Was hierunter genau $\mathrm{zu}$ verstehen ist, erwähnt er nicht. Er verdeutlicht aber, dass ihre Mitglieder über andere Eigenschaften verfügen sollten als diejenigen in der bürokratischen Verwaltung:

\section{Nur der Mann von edlem Gefühle findet in seinem eignen Herzen den Aufschluß uneigennütziger Handlungen, und der leidenschaftlichen Thätigkeit für das gemeine Wohl, die den meisten Menschen unbegreiflich ist und der deswegen so oft falsche Bewegungsgründe unterlegt werden. ${ }^{75}$}

Die Autoren der frühen Bürokratiekritik verstanden folglich 'Einfühlungsvermögen`, ’Kenntnisse lokaler Praktiken`, ‘Leidenschaft` und ‘Herzlichkeit` als Kriterien, die für die Einstellung von Staatsdienern zentral sein sollten. Diese Eigenschaften waren nach ihrer Auffassung unabdingbar, um notwendiges Vertrauen zu den Verwalteten aufzubauen und damit dem Gemeinwesen tatsächlich dienen zu können. Sie forderten demnach Spielräume für die Beamten, die genannten Eigenschaften zu entfalten, um damit das Gemeinwohl erhöhen zu können. Damit forderten sie eine (Re-)Integration von Praktiken, die den Amtsträgern die Möglichkeit gaben, auf Gefühlsbekundungen zu reagieren, ohne ihre eigene Ehre oder diejenige des Gegenübers negativ zu beeinflussen. Diese Forderung lief notwendigerweise auf die Re-Etablierung von Gabentauschpraktiken hinaus.

Der geschmähten Bürokratie setzten die Autoren zudem ein self government nach britischem Vorbild entgegen. Sie bezogen sich in ihren Ausführungen direkt und indirekt auf die Schriften Edmund Burkes. ${ }^{76}$ Jene wurden in den 1790er-Jahren von Friedrich von Gentz (1764-1832), einem engen Freund Adam Müllers, übersetzt und in Umlauf gebracht. Obwohl Burke sich in Großbritannien keineswegs einer vollausgebildeten Bürokratie gegenübersah, gelang es den Bürokratiekritikern, seine Argumentation auf die Verwaltungsreformen in den deutschen Staaten $\mathrm{zu}$ übertragen. ${ }^{77}$ Das self government, imaginiert als dezentrale Form des Verwaltens, korrespondierte mit der Rückbesinnung auf individuelle Ehre als Regulator und der Abkehr vom Berufsbeamtentum, die die 
Autoren wie gesehen einforderten. Insbesondere der Freiherr vom Stein spielte eine zentrale Rolle in diesem Zusammenhang, zumal sich auch einer der Autoren, nämlich Reisach, zu ihm in enger Beziehung befand.

Die Bürokratiekritiker mussten einigen Widerspruch aushalten. Zumal in Bayern erschienen Rechtfertigungsschriften, übrigens anonym verfasst von Reformbeamten, in denen die ebenerwähnten Eigenschaften nur zwei Zuschreibungen erhielten: Willkür und Missbrauch. Die Autoren griffen auf die Korruptionskritik wider das Ancien Régime zurück und versuchten zu verdeutlichen, dass erst das neue System »Ordnung und Klarheit" verschaffe ${ }^{78}$ und die »Mängel und Gebrechen des alten Lehenswesen « behoben habe. ${ }^{79}$ Zwar räumten sie Schwächen bei der Umsetzung des neuen Verwaltungssystems ein, insbesondere mit Blick auf die strenge Hierarchie, doch betonten sie dessen Wirksamkeit gegen Missbräuche. ${ }^{80}$ Die Forderung seitens der Bürokratiekritiker, den Spielraum für die Ausübung von Emotionspraktiken zu erweitern, deuteten sie als Versuch, korrupte Gabentauschsysteme, quasi ,über die Hintertürı, wiedereinzuführen.

Allein an dem Vorhandensein dieses Konflikts lässt sich erkennen, dass die Debatte längst nicht an ihr Ende gekommen war - im Gegenteil: Sie hatte gerade erst begonnen. In den nachfolgenden Jahren jedenfalls sollten die Kritiker der Bürokratisierung insofern an diskursivem Einfluss gewinnen, als ihre Kritik in vielen Zusammenhängen aufgegriffen und formuliert wurde. ${ }^{81}$ Die geringere Bedeutung des alten Ehrbegriffs konnten sie jedoch nicht verhindern; governance style und emotional style blieben von nun an getrennt.

\section{Fazit}

Im Zuge der bürokratischen Reformen im frühen 19. Jahrhundert entstand im Innern der Verwaltung ein ausgeprägtes Kontroll-, Etats- und Belegwesen, das von Amtsträgern als ein sinstitutionelles Misstrauen wahrgenommen wurde. Das Ziel des Artikels bestand darin zu zeigen, dass dieses sinstitutionelle Misstrauen einen Bruch mit den Emotionspraktiken der höheren Beamtenschaft darstellte, die ihr Verwaltungshandeln bis dahin bestimmten. Es widersprach dem Konzept aber vor allem durch Gabentauschpraktiken und Patron-Klient-Beziehungen zum Ausdruck kam. Letztere implizierten Emotionspraktiken im Sinne Monique Scheers, da sie Bekundungen $\mathrm{zu}$ >Treue`, 'Dankbarkeit`, >Pflichtgefühl etc. sowie praktische Reaktionen darauf provozierten und einem sozialen Handlungsschema folgten. Zwei Ebenen fanden Berücksichtigung: zum einen der inneradministrative Raum und zum anderen die publizistische Kritik an den neuen Verwaltungsstrukturen.

Die Fallbeispiele auf der inneradministrativen Ebene legten dabei offen, dass höhere Beamte ihre althergebrachten Praktiken beibehielten und so in einen Konflikt mit der Reformregierung um den althergebrachten emotional style gerieten. Gabentauschpraktiken mit Untergebenen und lokalen Eliten, die zuvor zum Selbstverständnis höherer Fürstendiener gehörten, standen dabei im Mittelpunkt. Mittels dieser Praktiken erhöhten und bezeugten Fürstendiener ihre persönliche Ehre und diejenige ihrer Familie. Ihre Ehre war zudem bestimmt von ihrem sozialen Rang, der auch in der Verwaltung eine Rolle spielte, und von der persönlichen Treuebeziehung zum Fürsten. Mit der Bürokratisierung ging eine Reihe einschneidender Neuerungen einher: Erstens verloren ständische Hierarchien inneradministrativ an Bedeutung, vor allem infolge der Abschaffung des Kollegialprinzips und der Etablierung verwaltungsinterner Hierarchien. Zweitens etablierte sich ein routinemäßsig eingerichtetes Revisions- und Kontrollwesen, infolge eines auf Etats basierenden Rechnungswesens. Drittens erfolgte eine Umcodierung bisher gestatteter Gabentausche: Die Beamten unterlagen einer Genehmigungspflicht, um Subordinierten Gratifikationen zu erteilen, es war ihnen untersagt, persönlich mit Verwaltungsexternen in Gabentauschbeziehungen einzutreten. Jede dieser Neuerungen provozierte Bekundungen eines verletzten Ehrgefühls vonseiten der höheren Staatsdiener: Genehmigungspflichten und Verbote kriminalisierten deren Gabentauschpraktiken und damit ihre Möglichkeiten, adäquat auf Gefühlsbekundungen von Verwaltungsinternen und -externen $\mathrm{zu}$ reagieren; das monokratische System konnte dazu führen, dass sozial gleichrangige oder gar niedergestellte Personen ihnen gegenüber weisungsbefugt 
waren; Untersuchungskommissionen suggerierten implizit Untreue gegenüber dem Fürsten. Diese Neuerungen hatten zugleich Konsequenzen für die Handlungsspielräume von Emotionspraktiken der höheren Beamtenschaft. Denn in dem persönlichen Wertehorizont der Akteure spielte symbolisches Kapital in Form von Ehre nach wie vor eine herausragende Rolle. Hielten sie sich an die Regeln, so war ihr Spielraum für Ehrerwerb und damit zugleich für Emotionspraktiken beschränkt. Behielten sie ihre Praktiken bei, nicht zuletzt deshalb, weil die Gegenseite etwa Untergebene und lokale Eliten - diese einforderte, so konnte es zu persönlichen Demütigungen durch das institutionelle Misstrauen ‘ kommen. Die Bürokratie verhinderte demnach die Ausübung eines althergebrachten emotional style auf die eine oder andere Weise, solange die Beamten ihr Verständnis von Ehre und den damit verbundenen Praktiken nicht aufgaben.

Diesen Konflikt um den emotional style griffen bürokratiekritische Publizisten auf. Sie schlussfolgerten, dass Staatsdiener in den neuen Strukturen zu gefühlskalten Kreaturen degenerieren würden, die nur nach ihrem Eigennutz streben. Die verwaltungsinternen Disziplinierungsmechanismen, in denen sich das institutionelle Misstrauen, manifestierte, seien letzten Endes wirkungslos. Sie würden nur mehr Ressourcen beanspruchen und den Verwaltungsapparat weiter aufblähen. In der Summe, so die Kritiker, führe das Verwaltungssystem zu einem moralischen - und finanziellen - Niedergang des Gemeinwesens. Deshalb sei es notwendig, Staatsdiener nicht primär aufgrund intellektueller Fähigkeiten auszuwählen. Vielmehr seien Tugend und Ehre die entscheidenden Rekrutierungskriterien. Ihre Voraussetzungen seien 'Einfühlungsvermögen‘, `Herzlichkeit` und andere sedle Gefühle`, die sie der stoten` und skalten` Bürokratie entgegenstellten. Damit rekurrierten sie zugleich auf jene Gefühlsbekundungen, die im Zusammenhang mit Gabentauschpraktiken standen, welche Staatsdiener im alltäglichen Geschäft weiter ausführten, aber inkommensurabel mit dem Grundwert der bürokratischen Verwaltung geworden waren, nämlich einem abstrakten Gemeinwohl zu dienen. Ihre Kritik lässt sich als Versuch deuten, die gemeinwohlschädlich $\mathrm{zu}$ delegitimieren. Zugleich versuchten sie damit, die althergebrachten Praktiken und die Autonomie höherer Staatsdiener zu legitimieren. Inwiefern auch diese Debatte zur Dichotomie emotional/ rational beitrug, die Verwaltung und Gesellschaft fortan prägte, muss an dieser Stelle offenbleiben. Es spricht aber einiges dafür.

Emotionsgeschichte, so ließen sich die Befunde verallgemeinern, kann von der historischen Korruptionsund Patronageforschung profitieren: Konflikte um korrupte Handlungen sind zumeist Aushandlungen über die Legitimität von Gabentauschpraktiken, welche zugleich ein Anschauungsobjekt für bestimmte Emotionspraktiken darstellen. Korruptionsdebatten sorgen nicht nur für kollektive Empörung im öffentlichen Raum, etwa bei großen Medienskandalen. ${ }^{82}$ Sie integrieren, jedenfalls in vielen Fällen, verbale Angriffe auf persönliche Nahbeziehungen und die Verteidigung der Letzteren. Gleiches gilt für die Patronageforschung, deren Protagonisten längst die damit verbundenen Gefühlsbekundungen thematisiert und problematisiert haben, zumal mit Blick auf

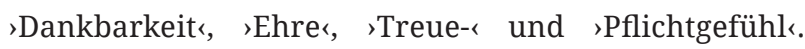
Korruptionskommunikation und -praktiken sowie Patronage übergreifend $\mathrm{zu}$ betrachten, gewährt Aufschluss über die Grenzen und Inhalte bestimmter emotional styles und kann, nämlich als Methode, zum Erkenntnisgewinn über deren historische Entwicklung beitragen.

Der Topos von der 'gefühlskalten oder sunmenschlichen` Bürokratie, um abschließend zum Ausgangspunkt zurückzukehren, lässt sich demnach bereits im frühen 19. Jahrhundert beobachten. Er war das Ergebnis eines Konfliktes um einen auf Gabentausch basierenden emotional style und enthielt bereits diejenigen Elemente, die wir heute kennen: gesichtslose Bürokraten, düstere Zukunftsprojektionen oder zahlenfixierte Leblosigkeit. 
1 So jedenfalls lauten aktuelle Urteile in Medien ganz unterschiedlicher Couleur in Deutschland, Frankreich oder dem Vereinigten Königreich, sei es bei der Bewertung der Europäischen Kommission oder des administrativen Umgangs mit Geflüchteten: Vgl. Philipp Woldin: "Erster Ausreisegewahrsam hat sogar ein Raucherzimmer «, online unter: https://www.welt.de/regionales/hamburg/article158961500/ Erster-Ausreisegewahrsam-hat-sogar-ein-Raucherzimmer.html (21. 10. 2016); vgl. Maxime Tandonnet: »La Commission européenne, un monstre froid et bureaucratique?«, online unter: http://www.lefigaro. fr/vox/politique/2014/05/22/31001-20140522ARTFIG00397-lacommission-europeenne-un-monstre-froid-et-bureaucratique.php (23. 5. 2014); vgl. Ruth Stark: »Refugee Children Arriving in the UK Need Warm Support - Not Cold Bureaucracy«, online unter: https:// www.theguardian.com/social-care-network/2016/oct/21/refugeechildren-uk-bureaucracy-social-work (21. 10. 2016).

2 Zu Kafkas Bürokratiekritik vgl. Burkhardt Wolf: »Kafka in Habsburg. Mythen und Effekte der Bürokratie«, in: Administory. Zeitschrift für Verwaltungsgeschichte 1 (2016), S. 193-221. Zur Bürokratiekritik bei The Wire vgl. Andrew Moore: "History, Freedom and Bureaucracy«, in: Arin Keeble / Ivan Stacy ( $\mathrm{Hg}$.): The Wire and America's Dark Corners. Critical Essays, Jefferson, North Carolina 2015, S. 13-30; vgl. David Simon: »Letter to HBO«, in: Rafael Alvarez/David Simon (Hg.): The Wire. Truth to Be Told, Edinburgh 2009, S. 32-36, hier S. 33.

3 Vgl. Karl Marx: "Zur Kritik der Hegelschen Rechtsphilosophie. Kritik des Hegelschen Staatsrechts«, in: Karl Marx/Friedrich Engels: Werke, Bd. 1, Berlin 1976, S. 203-333, hier S. 242-256.

4 Vgl. Max Weber: Wirtschaft und Gesellschaft. Grundriss der verstehenden Soziologie. Studienausgabe, hg. v. Johannes Winckelmann, Tübingen 51972 (EA 1921), S. 122-140, auch S. 551653. Zum Prozess vgl. Lutz Raphael: Recht und Ordnung. Herrschaft durch Verwaltung im 19. Jahrhundert, Frankfurt am Main 2000, S. 26-32, S. 76-93.

5 Vgl. Elisabeth Fehrenbach: Vom Ancien Régime zum Wiener Kongress, München 2010, S. 82-94, 109-125.

6 Vgl. Robert Bernsee: Moralische Erneuerung. Korruption und bürokratische Reformen in Bayern und Preußen, 1780-1820, Göttingen 2017, hier v. a. S. 71-161.

7 Vgl. ebd., S. 197-217.

8 Es gab Regeln, ihre Durchsetzung erfolgte jedoch nur selten. So war z. B. der Rentmeisterumritt ein Instrument der Kontrolle in Bayern, fand aber oft nur alle zehn bis zwanzig Jahre statt. Vgl. Helmut Rankl: »Der bayerische Rentmeister in der frühen Neuzeit. Generalkontrolleur der Finanzen und Justiz, Mittler zwischen Fürst und Bevölkerung, Promoter der sbaierischen Libertät«", in: Zeitschrift für bayerische Landesgeschichte 60 (1997), S. 617-648, hier S. 638.

9 Zum Problem der Definition von Emotionen vgl. Birgit Aschmann: "Vom Nutzen und Nachteil der Emotionen in der Geschichte. Eine Einführung«, in: dies. (Hg.): Gefühl und Kalkül. Der Einfluss von Emotionen auf die Politik des 19. und 20. Jahrhunderts, Stuttgart 2005, S. 9-32, hier S. 12-18; vgl. auch Jan Plamper: Geschichte und Gefühl. Grundlagen der Emotionsgeschichte, München 2012, S. 2034.

$10 \mathrm{Vgl}$. Monique Scheer: »Are emotions a kind of practice (and is that what makes them have a history)? A Bourdieuian approach to understanding emotion«, in: History and Theory 51 (2012), S. 193220. Scheers Zugriff richtet sich gegen die Dichotomie, die in der Forschung bislang dominierte. Demnach seien Emotionen entweder neurobiologische oder soziokulturelle Erscheinungen. Eine ähnliche Entwicklung, Emotionen übergreifend zu denken, scheint sich in der Psychologie etabliert zu haben: Vgl. Hilde Haider: »Emotionen als
Steuerungselemente menschlichen Handelns«, in: Birgit Aschmann (Hg.): Gefühl und Kalkül, S. 33-47.

11 Scheer: "Are emotions a kind of practice«, S. 193.

12 Vgl. ebd., S. 201-204.

13 Vgl. Pierre Bourdieu: „Ehre und Ehrgefühl«, in: ders.: Entwurf einer Theorie der Praxis auf der ethnologischen Grundlage der kabylischen Gesellschaft. Übersetzt von Cordula Pialoux und Bernd Schwibs, Frankfurt am Main 1979, S. 11-47, hier S. 21-23.

14 Vgl. Ronald G. Asch: "'Honour in all Parts of Europe will be ever like itself.، Ehre, adlige Standeskultur und Staatsbildung in England und Frankreich im 16. und 17. Jahrhundert: Disziplinierung oder Aushandlung von Statusansprüchen?«, in: Ronald G. Asch / Dagmar Freist (Hg.): Staatsbildung als kultureller Prozess. Strukturwandel und Legitimation von Herrschaft in der frühen Neuzeit, Köln/ Weimar/Wien 2005, S. 353-379; vgl. diverse Aufsätze in Sylvia Kesper-Biermann / Ulrike Ludwig / Alexandra Ortmann (Hg.): Ehre und Recht. Ehrkonzepte, Ehrverletzungen und Ehrverteidigungen vom späten Mittelalter bis zur Moderne, Magdeburg 2011; vgl. auch Ute Frevert: Ehrenmänner. Das Duell in der bürgerlichen Gesellschaft, München 1991.

15 Vgl. Sharon Kettering: "Gift-Giving and Patronage in Early Modern France», in: French History 2 (1988), S. 131-151. Es entstand gewissermaßen ein >Ethosı der Patronage: Vgl. Hillard von Thiessen: Diplomatie und Patronage. Die spanisch-römischen Beziehungen 1605-1621 in akteurszentrierter Perspektive, Epfendorf 2010, S. 382-385. Vgl. auch Birgit Emich: Bürokratie und Nepotismus unter Paul V. (1605-1621). Studien zur frühneuzeitlichen Mikropolitik in Rom, Stuttgart 2001, und - fast schon klassisch - Wolfgang Reinhard: „Freunde und Kreaturen. Historische Anthropologie von Patronage-Klientel-Beziehungen«, in: Freiburger Universitätsblätter 139 (1998), S. 127-141. Neuerdings für die Weimarer Republik: Volker Köhler: Genossen - Freunde - Junker: Mikropolitik personaler Beziehungen im politischen Handeln der Weimarer Republik, Göttingen 2018.

16 In diesem Sinne handelte es sich um eine Ökonomie der sEhres: Vgl. Andreas Pečar: Die Ökonomie der Ehre. Der höfische Adel am Kaiserhof Karls VI. (1711-1740), Darmstadt 2003, S. 20-140. Derlei Handlungen erfüllten damit Vertrauensfunktionen. Allein dadurch rücken sie in die Nähe jüngerer Forschungen der Emotionsgeschichte: Vgl. Ute Frevert: „Vertrauen. Historische Annäherungen an eine Gefühlshaltung«, in: Claudia Benthien / Anne Fleig / Ingrid Kasten (Hg.): Emotionalität. Zur Geschichte der Gefühle, Köln 2000, S. 178197; vgl. auch, mit zeithistorischem Bezug, diverse Aufsätze in Reinhild Kreis (Hg.): Diplomatie mit Gefühl. Vertrauen, Misstrauen und die Außenpolitik der Bundesrepublik Deutschland, Berlin 2015.

17 Vgl. Roland Mousnier: Les institutions de la France sous la monarchie absolue 1598-1789, Bd. 1: Société et Etat, Paris 1974, S. 85-93.

18 Vgl. Sharon Kettering: "Patronage in Early Modern France», in: French Historical Studies 17 (1992), S. 839-863.

19 Vgl. Heiko Droste: „Patronage in der Frühen Neuzeit - Institution und Kulturform«, in: Zeitschrift für historische Forschung 30 (2003), S. 555-590; vgl. Birgit Emich et al.: "Stand und Perspektiven der Patronageforschung. Zugleich eine Antwort auf Heiko Droste«, in: Zeitschrift für historische Forschung 32 (2005), S. 233-265.

20 Diese Komponenten von Emotionen orientieren sich an den Kategorien aufgeführt bei: Scheer: "Are emotions a kind of practice«, S. 209-217.

21 Vgl. ebd., S. 218.

22 Reinhart Koselleck rückte später vom Sattelzeitbegriff ab: Vgl. Reinhart Koselleck/Christof Dipper: "Begriffsgeschichte, Sozialgeschichte, begriffene Geschichte. Reinhard Koselleck im 
Gespräch mit Christof Dipper«, in: Neue Politische Literatur 43 (1998), S. 187-205, hier S. 194-196. In jüngeren Studien ist daher die Rede von 'Sattelzeit im Plural: Vgl. Jörn Leonhard: „Grundbegriffe und Sattelzeiten - Languages and Discourses. Europäische und anglo-amerikanische Deutungen des Verhältnisses von Sprache und Geschichte«, in: Rebekka Habermas (Hg.): Interkultureller Transfer und nationaler Eigensinn. Europäische und anglo-amerikanische Positionen der Kulturwissenschaften, Göttingen 2004, S. 71-86.

23 Vgl. Stefan Jordan: „Die Sattelzeit als Epoche«, in: Klaus E. Müller (Hrsg.): Historische Wendeprozesse. Ideen, die Geschichte machten, Freiburg 2003, S. 188-203.

24 Zur Entwicklung Bayerns in dieser Phase vgl. Walter Demel: Der bayerische Staatsabsolutismus 1806/08-1817. Staats- und gesellschaftspolitische Motivationen und Hintergründe der Reformära in der ersten Phase des Königreichs Bayern, München 1983; vgl. auch Hans-Peter Ullmann: Staatsschulden und Reformpolitik. Die Entstehung moderner öffentlicher Schulden in Bayern und Baden 1780-1820, Göttingen 1986.

25 Dementsprechend lautet das Urteil in etablierten Handbüchern: Vgl. Hans-Werner Hahn / Helmut Berding: Reformen, Restauration und Revolution. 1806-1848/49, Stuttgart 2010, S. 62-72; vgl. Walter Demel: Vom aufgeklärten Reformstaat zum bürokratischen Staatsabsolutismus, München 1993, S. 31-34.

26 Zur Ausgestaltung der Verwaltung frühneuzeitlicher Monarchien vgl. Wolfgang Reinhard: Geschichte der Staatsgewalt. Eine vergleichende Verfassungsgeschichte, München ${ }^{3} 2002$.

27 Vgl. Manfred Rauh: Verwaltung, Stände und Finanzen. Studien zu Staatsaufbau und Staatsentwicklung Bayerns unter dem späteren Absolutismus, München 1988, S. 14; vgl. Volker Press: „Die wittelsbachischen Territorien. Die pfälzischen Lande und Bayern«, in: Kurt G. A. Jeserich / Hans Pohl / Georg-Christoph Unruh (Hg.): Deutsche Verwaltungsgeschichte, Bd. 1: Vom Spätmittelalter bis zum Ende des Reiches, Stuttgart 1983, S. 552-599.

28 Dieser Umstand lässt sich vor allem an der Konstitution der Hausgesetze erkennen, in denen die Verfügungsgewalt der Fürsten - und dementsprechend der Familienoberhäupter - festgelegt wurde. Zu den Hausgesetzen allgemein vgl. Heinz Mohnhaupt: "Die Lehre von der >Lex Fundamentalisı und die Hausgesetzgebung europäischer Dynastien«, in: Heinz Mohnhaupt: Historische Vergleichung im Bereich von Staat und Recht. Gesammelte Aufsätze, Frankfurt am Main 2000, S. 1-33.

29 Darauf deuten die Aussagen in einzelnen Verordnungen etwa der 1780er hin, nach denen das Verhältnis zum Fürstendiener "eine bloße Gnaden- und willkürliche Sache« des Fürsten sei: Vgl. "Verordnung vom 23. 8. 1784, Nr. 54«, in: Georg Karl Mayr (Hg.): Sammlung der Kurpfalz-Baierischen allgemeinen und besonderen Landes-Verordnungen, Bd. 3: Von Justiz-, Finanz-, Landschafts-, Maut-, Accis-, Kommerzien-, Manufactur- oder Fabriquen-Sachen, München 1788, S. 312-314, hier S. 314.

30 Vgl. Rauh: Verwaltung, Stände und Finanzen, S. 73, 90. Ferner vgl. Stefan Brakensiek: Fürstendiener - Staatsbeamte - Bürger. Amtsführung und Lebenswelt der Ortsbeamten in niederhessischen Kleinstädten (1750-1830), Göttingen 1999, S. 159f; vgl. Angela Stirken: Der Herr und der Diener. Friedrich Carl von Moser und das Beamtenwessen seiner Zeit, Bonn 1984, S. 116-120.

31 Vgl.ThomasGross:Das KollegialprinzipinderVerwaltungsorganisation, Tübingen 1999, S. 111f. Zum Kollegialprinzip als typisches Phänomen frühneuzeitlicher Verwaltungen Europas vgl. Reinhard: Geschichte der Staatsgewalt, S. 171-179.

32 Vgl. dazu Birgit Näther: „Pragmatismus, Delegieren und Routinebildung. Zum Verhältnis vormoderner Verwaltungspraxis und Herrschaftsausübung«, in: Administory. Zeitschrift für Verwaltungswissenschaft 2 (2017), S. 35-48; vgl. Stefan Brakensiek: "Einleitung: Herrschaft und Verwaltung in der Frühen Neuzeit«, in: Stefan Brakensiek / Corinna von Bredow / Birgit Näther (Hg.): Herrschaft und Verwaltung in der Frühen Neuzeit, Berlin 2014, S. 9-24; Luise Schorn-Schütte: "Politische Kommunikation in der Frühen Neuzeit: Obrigkeitskritik im Alten Reich«, in: Geschichte und Gesellschaft 32 (2006), S. 273-314.

33 Vgl. Reinhard: Geschichte der Staatsgewalt, S. 132-140. Sie hatte damit eine ähnliche Funktion, wie sie Susanne Schattenberg für russische Beamte im 19. Jahrhundert beschrieben hat: Vgl. Susanne Schattenberg: „Die Ehre der Beamten oder: Warum die Staatsdiener nicht korrupt waren. Patronage in der russischen Provinzverwaltung im 19. Jahrhundert«, in: Jens Ivo Engels / Andreas Fahrmeir / Alexander Nützenadel (Hg.): Geld - Geschenke - Politik. Korruption im neuzeitlichen Europa, München 2009, S. 203-227; vgl. Susanne Schattenberg: Die korrupte Provinz? Russische Beamte im 19. Jahrhundert, Frankfurt am Main 2008.

34 Vgl. etwa Niels Grüne: "'Gabenschlucker und sverfreundte rätı. Zur patronagekritischen Dimension frühneuzeitlicher Korruptionskommunikation«, in: Ronald G. Asch et al. (Hg.): Integration - Legitimation - Korruption. Politische Patronage in Früher Neuzeit und Moderne, Frankfurt am Main 2011, S. 215-246; vgl. Ronald G. Asch: »Anfechtung und Legitimation. Beobachtungen zum Vergleich politischer Korruptionsdebatten in der Frühen Neuzeit», in: Niels Grüne / Simona Slanička (Hg.): Korruption. Historische Annäherungen an eine Grundfigur politischer Kommunikation, Göttingen 2010, S. 409-425.

35 Damit knüpft die Studie an die jüngere historische Korruptionsforschung an: Vgl. Jens Ivo Engels: Geschichte der Korruption. Von der Frühen Neuzeit bis ins 20. Jahrhundert, Frankfurt am Main 2014; vgl. Toon Kerkhoff: Hidden Morals, Explicit Scandals. Public Values and Political Corruption in the Netherlands (1748-1813), Diss., Leiden 2013; Christian Ebhardt: Interessenpolitik und Korruption. Personale Netzwerke und Korruptionsdebatten am Beispiel der Eisenbahnbranche in Großbritannien und Frankreich (11830-1870), Göttingen 2015; vgl. Ronald Kroeze / André Vitória / Guy Geltner (Hg.): Anti-Corruption in History. From Antiquity to the Modern Era, Oxford 2017.

36 Vgl. Bernd Wunder: Geschichte der Bürokratie in Deutschland, Frankfurt am Main 1986, S. 21-68; vgl. auch Reinhard Wendt: Die bayerische Konkursprüfung der Montgelas-Zeit. Einführung historische Wurzeln und Funktion eines wettbewerbsorientierten leistungsvergleichenden Staatsexamens, München 1984.

37 Vgl. Rainer Silbernagl: "Die Entwicklung der Systematik der Amtsdelikte und Gedanken zur Korruption im 18. und 19. Jahrhundert in der habsburgischen Gesetzgebung", in: Max Planck Institute for European Legal History Research Paper Series, No. 2017-09.

$38 \mathrm{Zu}$ den Veränderungen in der bayerischen Verwaltungsstruktur vgl. Wilhelm Volkert: »Bayern«, in: Kurt G. A. Jeserich / Hans Pohl / Georg-Christoph Unruh (Hg.): Deutsche Verwaltungsgeschichte. Bd. 2: Vom Reichsdeputationshauptschluß bis zur Auflösung des Deutschen Bundes, Stuttgart 1983, S. 503-550.

39 Vgl. Otto Rieder: "Karl August Graf von Reisach, der ehemalige Generalkommissär des Lech- und Illerkreises. Mit zwei Porträts. Hauptsächlich nach archivalischen, bisher unbenutzten Quellen bearbeitet", in: Oberbayerisches Archiv für vaterländische Geschichte 59 (1915), S. 189 -382, hier S. 191-207.

40 Es war in Bayern üblich, dass Pfleger ihr Amt auf ihre Nachkommen vererbten. Zuvor wurden derlei Ämter und andere käuflich erworben 
- u. a., um eigene Töchter zu einer sguten Partie`zu machen. Vgl. etwa Reinhard Wendt: Die bayerische Konkursprüfung, S. 21.

41 So jedenfalls die bisherige Forschung, etwa Otto Rieder, der dieses Handeln als "Prunksucht» begreift: Rieder: "Graf von Reisach«, S. 208.

42 Am Beispiel des preußischen Adels vgl. Carmen Winkel: Im Netz des Königs. Netzwerke und Patronage in der preußischen Armee 1713-1786, Paderborn 2013, S. 32; am Beispiel Englands vgl. Linda Levy Peck: Court Patronage and Corruption in Early Stuart England, Boston, Massachusetts 1990, S. 12-29.

43 Allerdings erfolgte 1806 ihre Auflösung. Vgl. Walter Demel: ")Revolution von oben`? Verfassungs- und Verwaltungsreformen in der Zeit des Aufgeklärten Absolutismus«, in: Michael Hochedlinger / Thomas Winkelbauer (Hg.): Herrschaftsverdichtung, Staatsbildung, Bürokratisierung. Verfassungs-, Verwaltungs- und Behördengeschichte der Frühen Neuzeit, Wien 2010, S. 213-228, hier S. $218 f$.

44 Zur Korruptionskritik in Deutschland im späten 18. und frühen 19. Jahrhundert vgl. Robert Bernsee: "Corruption in German Political Discourse between 1780 and 1820: A Categorisation«, in: Journal of Modern European History 11 (2013), S. 54-73.

45 Vgl. dazu Margot Hamm: Die bayerische Integrationspolitik in Tirol 1806-1814, München 1996, S. 312-320.

46 Vgl. Rieder: „Graf von Reisach«, S. $233 f$.

47 "Brief von Reisach an Montgelas vom 21. 6. 1808«, in: BHStA MInn 44799, 2vr.

48 Vgl. Rieder: "Graf von Reisach«, S. 291-319.

49 »Brief von Joseph von Stichaner an Montgelas vom 13. 7. 1815«, in: BHStA MInn 43560, unfoliiert.

50 »Brief von Gropper an Montgelas vom 16. 6. 1815«, in: BHStA MInn 43560, unfoliiert.

51 Vgl. »Brief von Montgelas an Gropper vom 5. 8. 1815«, in: BHStA MInn 43560, unfoliiert.

52 Vgl. etwa Heinz Duchhardt: »Das diplomatische Abschiedsgeschenk. Herrn Prof. Dr. Eberhard Kessel zum 70. Geburtstag gewidmet«, in: Archiv für Kulturgeschichte 57 (1975), S. 345-362.

53 Florian Kühnel etwa betont, dass (drohender) Ehrverlust zu Selbsttötungen unter Adeligen führen konnte. Vgl. Florian Kühnel: Kranke Ehre? Adlige Selbsttötung im Übergang zur Moderne, München 2013, S. 312-314.

54 Vorgang dokumentiert in: BHStA MA 39404, unfoliiert.

55 Vgl. BHStA, MF 2287, fol. 3.

56 Zur Geschichte der frühen Bürokratiekritik vgl. auch Pascale Cancik: „Zuviel Staat? - Die Institutionalisierung der `Bürokratie`-Kritik im 20. Jahrhundert«, in: Der Staat. Zeitschrift für Staatslehre und Verfassungsgeschichte, deutsches und europäisches öffentliches Recht 56 (2017), S. 1-38, hier vor allem S. 3-5.

57 Vgl. Barbara Stollberg-Rilinger: Der Staat als Maschine. Zur politischen Metaphorik des absoluten Fürstenstaats, Berlin 1986, S. 203-216.

58 August Wilhelm Rehberg: Ueber die Staatsverwaltung deutscher Länder und die Dienerschaft des Regenten, Hannover 1807, S. 49.

59 Ebd., S. 81.

60 Adam Heinrich Müller: Die Elemente der Staatskunst, 1. Theil, Berlin 1809, S. 3; vgl. ders.: Die Elemente der Staatskunst, 2. Theil, Berlin 1809, S. 195.

61 Diese Bezeichnung wählt ein anderer bürokratiekritischer Autor, nämlich Ernst Moritz Arndt: Ueber künftige ständische Verfassungen in Teutschland, s. I. 1814, S. 25.

62 Rehberg: Ueber die Staatsverwaltung, S. 12

63 Ebd., S. 52
64 Ernst von Bülow-Cummerow: Ueber die Verwaltung des Staatskanzlers Fürsten von Hardenberg. Fortsetzung der Schrift: Ein Punkt auf's I, Zerbst 1821, S. 90.

65 In Preußen etwa, woher Bülow kam, wuchs bis 1850 die Bevölkerung durchschnittlich um gut 1,3 Prozent per annum, während das Personal in der Administration nur um knapp 0,2 Prozent zunahm. Vgl. Hansjoachim Henning: Die deutsche Beamtenschaft im 19. Jahrhundert. Zwischen Stand und Beruf, Stuttgart 1984, S. 33.

66 Zu Stein während dieser Zeit vgl. Heinz Duchhardt: Stein. Eine Biographie, Münster 2007, S. 261-324.

67 Zur Etablierung dieser pressepolitischen Instrumente vgl. Wolfgang Piereth: Bayerns Pressepolitik und die Neuordnung Deutschlands nach den Befreiungskriegen, München 1999, passim.

68 Karl August von Reisach: Der Graf Karl August von Reisach-Steinberg an das teutsche Volk, Teutschland 1814, S. 51.

69 Vgl. Müller: Die Elemente, 2. Theil, S. 123.

70 Zur frühen Chronologie der amtlichen Statistik in Bayern vgl. Bayerischen Landesamt für Statistik und Datenverarbeitung (Hrsg.): 200 Jahre amtliche Statistik in Bayern 1808 bis 2008. München 2008, S. 9-13. Über Entwicklung des statistischen Diskurses in Bayern im späten 18. Jahrhundert vgl. Lars Behrisch: Die Berechnung der Glückseligkeit. Statistik und Politik in Deutschland und Frankreich im späten Ancien Régime, Ostfildern 2016, S. 193-316.

71 Rehberg: Ueber die Staatsverwaltung, S. 14.

72 Vgl. [Karl August von Reisach]: Baiern unter der Regierung des Ministers Montgelas, [Landshut] 1813, S. $35 f$.

73 Bülow: Verwaltung, S. 94.

74 Rehberg: Ueber die Staatsverwaltung, S. 159

75 Ebd., S. 107.

76 Beispielhaft: Vgl. Müller: Die Elemente, Theil 1, S. 26, 29-33; vgl. Reisach: Baiern unter der Regierung, S. 50.

77 Zur frühen Rezeption Edmund Burkes in Deutschland: Vgl. László Kontler: »The Ancien Régime in Memory and Theory. Edmund Burke and his German Followers", in: European Review of History 4 (1997), S. 31-43.

78 Christoph von Aretin: Abhandlungen über wichtige Gegenstände der Staatsverfassung und Staatsverwaltung mit besonderer Rücksicht auf Bayern, München 1816, S. 155.

79 Ebd., S. 150.

80 Vgl. ebd., S. 86.

81 Vgl. Cancik: "Zuviel Staat«, S. 5-7; vgl. auch Raphael: Recht und Ordnung, S. 194-198.

82 Vgl. dazu etwa Frank Bösch: Öffentliche Geheimnisse. Skandale, Politik und Medien in Deutschland und Großbritannien 1880-1914, München 2009, S. 421-468. 


\section{Abstract}

The article deals with the narrative of the 'cold' or 'inhumane bureaucracy'. The author argues that one can already observe this narrative during the early bureaucratisation in Germany. He shows that the perception of a 'cold bureaucracy' resulted from conflicts about the legitimacy of administrative practices: The new bureaucratic system clashes with the traditional practices exercised by officials, among them certain gift-giving practices. Those actions were based on a concept of honour, they shaped the emotional practices of the officials. The bureaucratic system reduced the spaces for those emotional practices, because it interdicted traditional practices. Officials argued that this interdiction and the bureaucratic instruments of monitoring and controlling was mistrusting and defamatory to them. Public observers condemned the new administrative system to be 'cold' and 'inhumane'. They demanded more spaces for emotional practices by reallowing traditional actions and re-introducing elements of the old administrative system.

\section{About the Author}

Robert Bernsee is a research associate and lecturer at the Institute for Economic and Social History at University of Goettingen in Germany. His research has focused on the history of corruption, ethics and governance in Germany around 1800. He currently conducts historical research on whistle-blowing and on copyright regimes in Germany and the United States during the twentieth century. Bernsee has recently published a book on corruption in Bavaria and Prussia during the Sattelzeit (Moralische Erneuerung. Korruption und bürokratische Reformen in Bayern und Preußen, 1780-1820, Göttingen 2017). 